\title{
A general, ring closure metathesis based enantiospecific approach to polyfunctional eudesmane, eremophilane and agarofuran sesquiterpenoids
}

\author{
Goverdhan Mehta* and R. Senthil Kumaran \\ Department of Organic Chemistry, Indian Institute of Science, Bangalore 560 012, India
}

\begin{abstract}
An enantiospecific and stereo- and functional group diversity oriented approach to eudesmane, eremophilane and agarofuran sesquiterpenoids from (-)-carvone has been devised. RCM has been employed as the key reaction to generate the highly functionalized eudesmane framework. Further elaboration of the eudesmane framework to agarofurans and biogenetic-type rearrangement to eremophilanes is outlined.
\end{abstract}

Eudesmane $\mathbf{1}$ and eremophilane $\mathbf{2}$ sesquiterpenoid skeleta based on the bicyclic decalin framework are fairly widespread in nature and occur in different stereochemical and functionalization patterns. ${ }^{1}$ Eremophilane 2 represents the earliest known example of an irregular isoprenoid in nature and in a classical proposal, Robinson in $1939^{2}$ invoked a carbonium ion-mediated methyl migration in eudesmane 1 to explain their biogenesis which was subsequently confirmed through both in vivo and in vitro experiments. ${ }^{3}$ Another interesting sub-class of natural products derived from eudesmanes are agarofurans $\mathbf{3}$ whose polyoxygenated derivatives, e.g. celorbicol $\mathbf{4}$ are of wide occurrence, particularly in the Celastraceae family and many of them have been found to exhibit broad ranging biological activity profiles. ${ }^{4,5}$ Not surprisingly, eudesmanes, eremophilanes and particularly agarofurans continue to evoke a great deal of synthetic interest and many interesting strategies have been devised to access these families of sesquiterpenoids. ${ }^{1,6}$ In particular, extensive synthetic studies have been directed towards the polyhydroxylated agarofurans in view of their biological potential and the challenge associated with the generation of the network of hydroxyl functionalities. ${ }^{6}$ Herein, we describe a new, general, enantiospecific synthetic approach towards eudesmanes from (-)-carvone $\mathbf{5}$ employing a ring closing metathesis (RCM) reaction ${ }^{7}$ as the key step and their further elaboration towards

* Corresponding author. E-mail: gm@orgchem.iisc.ernet.in polyfunctional agarofurans as well as biogenetic-type rearrangement to the eremophilane framework.

The $\alpha, \beta$-epoxyketone $6,{ }^{8}$ readily obtained from (-)-carvone 5 , on reductive allylation under dissolving metal conditions charted an interesting course and epimeric hydroxy-ketones $\mathbf{7}$ and $\mathbf{8}$ (45:55), sharing identical stereo- chemistry at the newly generated quaternary center were realized (Scheme 1). ${ }^{9}$ The epimeric nature of the hydroxy-ketones followed from their oxidation to the same dione 9 and their formation involving an eventful fragmentation-realdolization process is depicted in Scheme 1. It is worth noting that the allyl group in $\mathbf{7}$ and $\mathbf{8}$ is $\mathrm{cis}$ with respect to the isopropenyl group, a situation conducive to accessing eudesmane based natural products which occur predominantly with the angular methyl group and the isopropyl group in a trans arrangement.

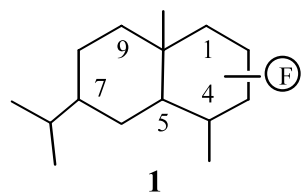<smiles>CC(C)C1CCC2CCCCC2(C)C1(C)C</smiles>

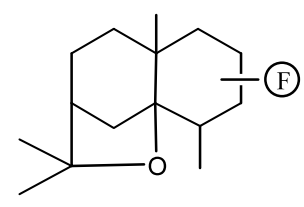

3

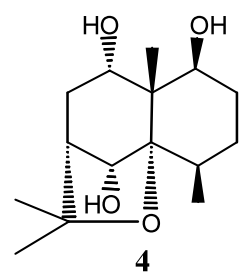



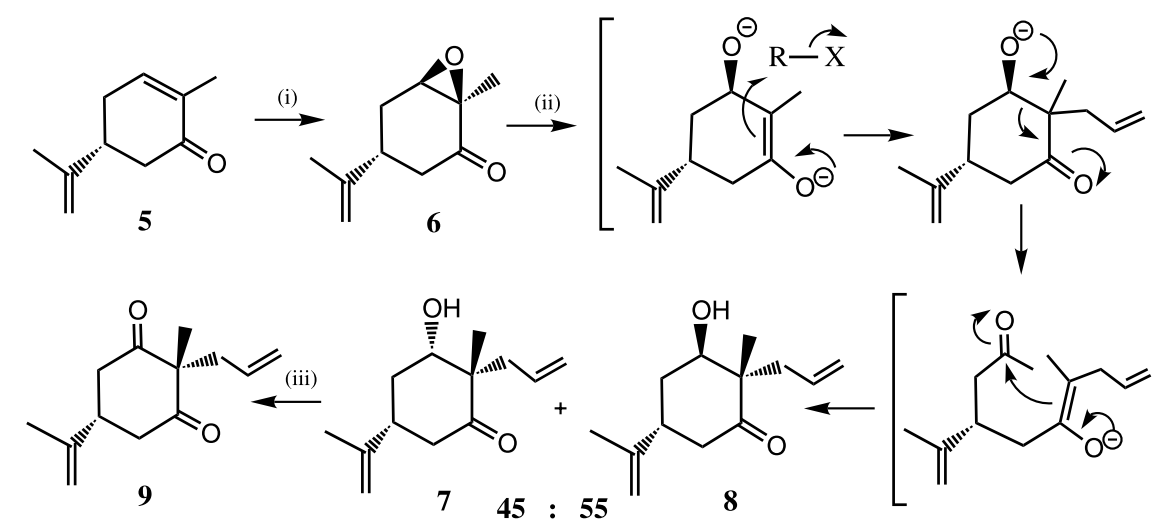

Scheme 1. Reagents and conditions: (i) $30 \% \mathrm{H}_{2} \mathrm{O}_{2}, 6 \mathrm{~N} \mathrm{NaOH}, \mathrm{MeOH}, 0{ }^{\circ} \mathrm{C}, 92 \%$; (ii) Li, liq. $\mathrm{NH}_{3}, \mathrm{CH}_{2}=\mathrm{CHCH}_{2} \mathrm{Br} \mathrm{Et}_{2} \mathrm{O},-78^{\circ} \mathrm{C}$, 60\%; (iii) PCC, DCM, 88\%.

Addition of the Grignard reagent derived from 3chloro-1-butene to the major $\beta$-hydroxy-ketone $\mathbf{8}$ in the presence of $\mathrm{Ce}(\mathrm{III})$ furnished two diastereomeric addition products $\mathbf{1 0}$ and $\mathbf{1 1}$ (55:45) (Scheme 2). Interestingly, the Grignard reagent addition to the carbonyl group of $\mathbf{8}$ was $\alpha$-face selective to furnish $\mathbf{1 0}$ and $\mathbf{1 1}$ with the two allyl side arms in cis disposition. Both $\mathbf{1 0}$ and $\mathbf{1 1}$ were now set for the RCM reaction to generate the eudesmane framework. Indeed, exposure of $\mathbf{1 0}$ and 11 to Grubbs' catalyst [benzylidene-bis(tricyclohexylphosphine)-dichlororuthenium] resulted in smooth and efficient conversion to bicycles 12 and 13, respectively (Scheme 2) ${ }^{9}$ and their stereostructures were secured through the X-ray crystal structure determination of the PNB-derivative of $\mathbf{1 2} .^{10}$ Thus, in a short sequence from carvone 5, highly functionalized eudesmanes 12 and 13 could be accessed, paving the way for further transformations.

Polyfunctional dihydroxyeudesmane $\mathbf{1 3}$ was well-poised for further elaboration to the eremophilane ring system 2. The monobenzoate $\mathbf{1 4}$ derived from $\mathbf{1 3}$ was smoothly and regioselectively dehydrated to the bicyclic triene $\mathbf{1 5}$ (Scheme 3). ${ }^{9}$ The exocyclic double bond in $\mathbf{1 5}$ could be regioselectively hydrated through oxymercuration to furnish the tertiary alcohol $\mathbf{1 6} .^{9}$ Further epoxidation of 16 was stereoselective but exhibited only 4:1 regioselectivity and a 4:1 mixture of $\alpha$-epoxides 17 and $\mathbf{1 8}$ was obtained (Scheme 3). Exposure of the major epoxide 17 to $\mathrm{BF}_{3}$-etherate and hydrolysis of the intermediate enolbenzoate 19, formed through sequential methyl and hydrogen migrations, resulted in the eremophilane keto-diol 20 in good yield (Scheme 3). ${ }^{9}$ The olefinic moiety in $\mathbf{2 0}$ was further hydroxylated with $\mathrm{OsO}_{4}$ to furnish polyfunctional eremophilane $\mathbf{2 1}$ whose stereostructure was secured through single crystal X-ray structure determination. ${ }^{10}$

As per our plan, dihydroxyeudesmane derivative $\mathbf{1 3}$ appeared to be serviceable for elaboration to the agarofuran framework 3, particularly because the $\mathrm{C} 4 \beta$ methyl stereochemistry present in it is widespread among natural products of this class. However, in the biologically more important agarofurans, the C9 hydroxyl group is usually $\alpha$-oriented, see celorbicol $\mathbf{4}$. Our first effort therefore was to carry out a Mitsunobu type reaction on $\mathbf{1 3}$ to invert the $\mathrm{C} 9$ hydroxy group stereochemistry but these efforts were not successful. Consequently, 13 was oxidised to ketone 22 (X-ray crystal structure) ${ }^{10}$ and its reduction with sodium boro-

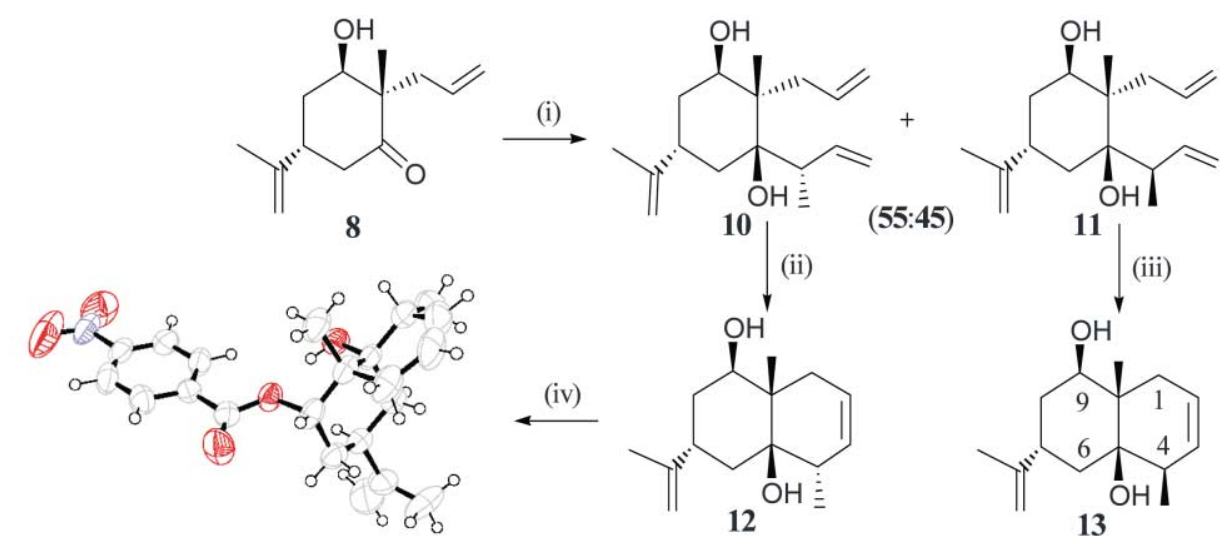

ORTEP diagram of the PNB derivative of 12

Scheme 2. Reagents and conditions: (i) $\mathrm{CH}_{2}=\mathrm{CHCH}\left(\mathrm{CH}_{3}\right) \mathrm{MgCl}$, anhyd. $\mathrm{CeCl}_{3}, \mathrm{THF},-78^{\circ} \mathrm{C}, 95 \%$; (ii) $\mathrm{Grubbs}$ ' cat. (7 mol\%), $\mathrm{C}_{6} \mathrm{H}_{6}, 85^{\circ} \mathrm{C}, 89 \%$; (iii) Grubbs' cat. (5 mol\%), $\mathrm{C}_{6} \mathrm{H}_{6}, 85^{\circ} \mathrm{C}, 95 \%$; (iv) $p-\mathrm{NO}_{2} \mathrm{C}_{6} \mathrm{H}_{4} \mathrm{COCl}, \mathrm{Et}{ }_{3} \mathrm{~N}, \mathrm{DCM}, \mathrm{rt}, 90 \%$. 
<smiles>C=C(C)[C@@H]1C[C@@H](O)[C@]2(C)CC=C[C@H](C)[C@]2(O)C1</smiles>

13

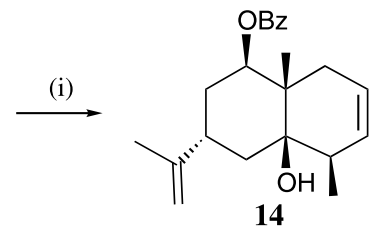

(4: 1)<smiles>C[C@@H]1C2=C[C@H](C(C)(C)O)C[C@H](C(C)(C)C)[C@]2(C)C[C@H]2O[C@@H]21</smiles>

(v)<smiles>CC1CC1(O)[C@H]1CC(OC(C)(C)C)=C2CC=C[C@H](C)[C@@]2(C)[C@@H]1O</smiles>

19<smiles>CC#CC(C)(C)[C@@H]1CC(=O)[C@H]2CC=C[C@H](C)[C@]2(C)[C@H]1O</smiles>

20 (iv)<smiles>C[C@H]1C=CC[C@]2(C(=O)OCc3ccccc3)C1=C[C@H](C(C)(C)O)C[C@@H]2OC(C)(C)C</smiles>

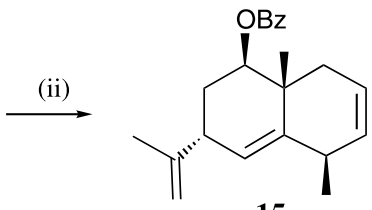

15<smiles></smiles>

$\stackrel{\text { (vii) }}{\longrightarrow}$<smiles>C[C@H]1[C@@H](O)[C@H](O)C[C@H]2C(=O)C[C@H](C(C)(C)O)[C@@H](O)[C@@]21C</smiles>

21

Scheme 3. Reagents and conditions: (i) $\mathrm{C}_{6} \mathrm{H}_{5} \mathrm{COCl}, \mathrm{Et}_{3} \mathrm{~N}$, DMAP, DCM, rt, $90 \%$; (ii) $\mathrm{SOCl}_{2}$, pyridine, $-35^{\circ} \mathrm{C}, 85^{\circ}$; (iii) $\mathrm{Hg}(\mathrm{OAc})_{2}$, cat. $\mathrm{AcOH}$, THF: $\mathrm{H}_{2} \mathrm{O}$, rt, 75\%; (iv) $m \mathrm{CPBA}, \mathrm{NaHCO}_{3}, \mathrm{DCM}, 83 \%$; (v) $\mathrm{BF}_{3} \cdot \mathrm{Et}_{2} \mathrm{O}, \mathrm{DCM}, 0{ }^{\circ} \mathrm{C}, 80 \%$; (vi) $\mathrm{K}_{2} \mathrm{CO}_{3}, \mathrm{MeOH}^{\circ}, \mathrm{rt}, 95 \%$; (vii) $\mathrm{OsO}_{4}, \mathrm{NMMO}, \mathrm{CH}_{3} \mathrm{COCH}_{3}: \mathrm{H}_{2} \mathrm{O}, \mathrm{rt}, 92 \%$.

hydride led to a readily separable 4:1 mixture of $\mathbf{1 3}$ and the epimeric diol 23. Although the stereoselectivity in this reaction to access desired $\mathbf{2 3}$ was not satisfactory, 13 could be recycled via 22 to build reasonable quantities of 23 (Scheme 4). ${ }^{9}$ Monobenzoate $\mathbf{2 4}$ derived from
23 was dehydrated to furnish triene $\mathbf{2 5}$ regioselectively (Scheme 4). Oxymercuration of $\mathbf{2 5}$ was regioselective at the terminal olefinic moiety and readily led to the tertiary alcohol 26. ${ }^{9}$ Catalytic $\mathrm{OsO}_{4}$-mediated dihydroxylation in $\mathbf{2 6}$ was stereoselective and acetonide protec-<smiles>C=C(C)[C@@H]1C[C@@H](O)[C@]2(C)CC=CC(C)[C@]2(O)C1</smiles>

13<smiles>CC1C=CC[C@@]2(C)C1=C[C@H](C(C)(C)C)[C@H](C(=O)O)C[C@H]2O</smiles>

$\mathrm{OH} 26$

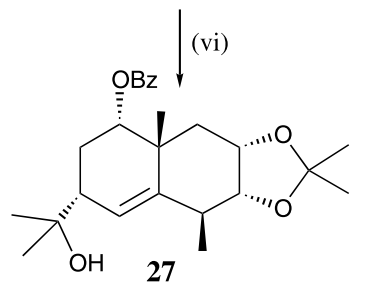

(v)<smiles>C=C(C)[C@@H]1C=C2[C@H](C)C=CC[C@]2(C)[C@H](O)C1</smiles>

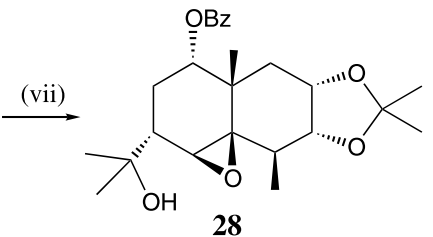

(iv)

$\stackrel{\text { (ii) }}{\longrightarrow}$<smiles>C=C(C)[C@@H]1C[C@@H](O)[C@]2(C)CC=[C+][C@H]([14CH3])[C@]2(O)C1</smiles>

$\stackrel{\text { (viii) }}{\longrightarrow}$<smiles>CC1[C@H](O)[C@@H](O)C[C@@]2(C)[C@@H](O)CC[C@@H](C(C)(C)C)[C@@]12O</smiles>

Scheme 4. Reagents and conditions: (i) $\mathrm{PCC}, \mathrm{DCM}, \mathrm{rt}, 78 \%$; (ii) $\mathrm{NaBH}_{4}, \mathrm{MeOH}, 0^{\circ} \mathrm{C}, 90 \%$; (iii) $\mathrm{C}_{6} \mathrm{H}_{5} \mathrm{COCl}, \mathrm{Et}_{3} \mathrm{~N}, \mathrm{DMAP}, \mathrm{DCM}$, rt, 87\%; (iv) $\mathrm{SOCl}_{2}$, pyridine, $-35^{\circ} \mathrm{C}, 80 \%$; (v) $\mathrm{Hg}(\mathrm{OAc})_{2}$, cat. $\mathrm{AcOH}, \mathrm{THF}: \mathrm{H}_{2} \mathrm{O}, \mathrm{rt}, 70 \%$; (vi) (a) OsO ${ }_{4}, \mathrm{NMMO}$, $\mathrm{CH}_{3} \mathrm{COCH}_{3}: \mathrm{H}_{2} \mathrm{O}$, rt, 95\%; (b) $\mathrm{CH}_{3} \mathrm{COCH}_{3}$, Amberlyst, rt, 81\%; (vii) $m \mathrm{CPBA}, \mathrm{NaHCO}_{3}, \mathrm{DCM}, 90 \%$; (viii) TMSOTf, DCM, $0^{\circ} \mathrm{C}$, $55 \%$. 
tion led to the polyfunctional 27 (Scheme 4). As the $\alpha$-face of 27 was sterically shielded, epoxidation of $\mathbf{2 7}$ was stereoselective and furnished exclusively the $\beta$ epoxide 28. Brief exposure of $\mathbf{2 8}$ to TMSOTf led to the contemplated tetrahydrofuran formation through epoxide opening and capture by the tertiary hydroxyl group to lead to agarofuran derivative 29. The complete stereostructure of $\mathbf{2 9}$ was confirmed through its X-ray crystal structure determination. ${ }^{10}$ Access to the agarofuran 29 with secured stereochemistry at eight stereogenic centers and five oxygen functionalities, particularly with C6 and C9 hydroxyl groups, makes our approach amenable to adaptation for the synthesis of many natural products of this family.

In summary, we have outlined a new approach to assemble rapidly, strategically functionalized eudesmanes from (-)-carvone employing RCM as the key step. Further transformations involving functional group amplification and rearrangements provide entry into polyoxyfunctionalized eremophilanes and agarofurans.

\section{Acknowledgements}

We thank JNCASR for the financial support. One of us (R.S.K.) thanks CSIR for the award of a research fellowship.

\section{References}

1. Reviews: (a) Fraga, B. M. Nat. Prod. Rep. 2002, 19, 650-672 and earlier contributions by the same author in this review series. (b) Faulkner, D. J. Nat. Prod. Rep. 2002, 19, 1-48 and earlier contributions by the same author in this review series.

2. (a) Cited in: Penfold, A. R.; Simonsen, J. L. J. Chem. Soc. 1939, 87-89; (b) Robinson, R. Structural Relations of Natural Products; Oxford University Press: London, 1955; p. 12.

3. (a) Miller, C. A.; Pinder, A. R. J. Chem. Soc., Chem. Commun. 1977, 230-231; (b) Ceccherelli, P.; Curini, P.; Marcotullio, M. C.; Rosati, O. Tetrahedron 1989, 45, 3809-3818.

4. For a recent review, see: Spivey, A. C.; Weston, M.; Woodhead, S. Chem. Soc. Rev. 2002, 31, 43-59.

5. Selected examples of agarofuran natural products, see: (a) Maheshwari, M. L.; Jain, T. C.; Bates, R. B.; Bhattacharyya, S. C. Tetrahedron 1963, 19, 1079-1090; (b) Maheshwari, M. L.; Varma, K. R.; Bhattacharyya, S. C. Tetrahedron 1963, 19, 1519-1525; (c) Bruning, R.; Wagner, H. Phytochemistry 1978, 17, 1821-1858; (d) Kim, S. E.; Kim, Y. H.; Lee, J. J. J. Nat. Prod. 1998, 61, 108-111.

6. For some recent references towards the synthesis of agarofurans: (a) White, J. D.; Shin, H.; Kim, T.-S.; Cutshall, N. S. J. Am. Chem. Soc. 1997, 119, 2404-2419; (b) Tu, Y. Q.; Sun, L. D. Tetrahedron Lett. 1998, 39, 7935-7938; (c) Zhou, G.; Gao, X.; Li, W. Z.; Li, Y. Tetrahedron Lett. 2001, 42, 3101-3103 and earlier contributions of this group; (d) Spivey, A. C.; Woodhead, S. J.;
Weston, M.; Andrews, B. I. Angew. Chem., Int. Ed. 2001, 40, 769-771; (e) Xia, W. J.; Li, D. R.; Shi, L.; Tu, Y. Q. Tetrahedron Lett. 2002, 43, 627-630; (f) Boyer, F.-D.; Descoins, C. L.; Descoins, C.; Prange, T.; Ducrot, P.-H. Tetrahedron Lett. 2002, 43, 8277-8279 and earlier papers by this group.

7. (a) Grubbs, R. H.; Chang, H. Tetrahedron 1998, 54, 4413-4450; (b) Armstrong, S. K. J. Chem. Soc., Perkin Trans. 1 1998, 371-388.

8. (a) McChesney, J. D.; Wycpalek, A. F. J. Chem. Soc., Chem. Commun. 1971, 542; (b) Szajewski, R. P. J. Org. Chem. 1978, 43, 1819-1820.

9. All new compounds reported here were duly characterized on the basis of spectroscopic data (IR, ${ }^{1} \mathrm{H}$ and ${ }^{13} \mathrm{C}$ NMR) and elemental analyses. Data for 29: mp 213$214^{\circ} \mathrm{C} ;[\alpha]_{\mathrm{D}}=-11.1\left(c 0.45, \mathrm{CHCl}_{3}\right)$, IR $\left(\mathrm{cm}^{-1}\right) 3438$, 1711; ${ }^{1} \mathrm{H}$ NMR $\left(300 \mathrm{MHz}, \mathrm{CDCl}_{3}\right) \delta 8.06(\mathrm{~d}, J=7.2 \mathrm{~Hz}$, $2 \mathrm{H}), 7.58(\mathrm{t}, J=7.5 \mathrm{~Hz}, 1 \mathrm{H}), 7.47(\mathrm{t}, J=7.5 \mathrm{~Hz}, 2 \mathrm{H}), 5.10$ $(\mathrm{d}, J=7.5 \mathrm{~Hz}, 1 \mathrm{H}), 4.70$ (br s, $1 \mathrm{H}), 4.30$ (d, $J=9.9 \mathrm{~Hz}$, $1 \mathrm{H}), 3.91$ (br s, $-\mathrm{OH}), 3.69$ (d, $J=9.6 \mathrm{~Hz}, 1 \mathrm{H}), 2.71$ (ddd, $J=16.5,7.5,4.2 \mathrm{~Hz}, 1 \mathrm{H}$ ), 2.46-2.43 (br m, -OH), 2.36$2.20(\mathrm{~m}, 2 \mathrm{H}), 1.99-1.93(\mathrm{~m}, 2 \mathrm{H}), 1.79$ (br s, $-\mathrm{OH}), 1.48$ (s, $6 \mathrm{H}), 1.32(\mathrm{~s}, 3 \mathrm{H}), 1.31(\mathrm{~d}, J=7.8 \mathrm{~Hz}, 3 \mathrm{H}), 1.17(\mathrm{dd}$, $J=12.9,4.5 \mathrm{~Hz}, 1 \mathrm{H}) ;{ }^{13} \mathrm{C} \mathrm{NMR}(75 \mathrm{MHz}) \delta 166.1,132.9$, $130.4,129.7,128.5,83.5,79.7,76.9,75.9,75.8,65.9,47.8$, 46.9, 45.4, 35.8, 30.6, 25.5, 25.3, 25.1, 14.9; HRMS calcd for $413.1940(\mathrm{M}+\mathrm{Na})$. Found: 413.1945. 28: $[\alpha]_{\mathrm{D}}=-19.0$ (c 1.0, $\left.\mathrm{CHCl}_{3}\right)$; IR $\left(\mathrm{cm}^{-1}\right) 3452,1718,1273 ;{ }^{1} \mathrm{H}$ NMR $\left(300 \mathrm{MHz}, \mathrm{CDCl}_{3}\right) \delta 8.03(\mathrm{~d}, J=7.8 \mathrm{~Hz}, 2 \mathrm{H}), 7.57(\mathrm{t}$, $J=7.8 \mathrm{~Hz}, 1 \mathrm{H}), 7.45(\mathrm{t}, J=7.8 \mathrm{~Hz}, 2 \mathrm{H}), 4.96(\mathrm{dd}, J=$ $11.7,3.6 \mathrm{~Hz}, 1 \mathrm{H}), 4.51-4.44(\mathrm{~m}, 1 \mathrm{H}), 4.18-4.16(\mathrm{~m}, 1 \mathrm{H})$, $3.13(\mathrm{~s}, 1 \mathrm{H}), 2.19-1.92(\mathrm{~m}, 4 \mathrm{H}), 1.77-1.73(\mathrm{~m}, 2 \mathrm{H}), 1.52$ $(\mathrm{s}, 3 \mathrm{H}), 1.37(\mathrm{~s}, 3 \mathrm{H}), 1.31(\mathrm{~s}, 3 \mathrm{H}), 1.30(\mathrm{~s}, 3 \mathrm{H}), 1.22(\mathrm{~d}$, $J=7.8 \mathrm{~Hz}, 3 \mathrm{H}), 1.15(\mathrm{~s}, 3 \mathrm{H}) ;{ }^{13} \mathrm{C} \mathrm{NMR}(75 \mathrm{MHz}) \delta$ $165.9,133.1,130.2,129.6,128.4,107.9,79.5,75.9,72.1$, $71.3,65.4,64.2,44.8,38.8,37.6,32.3,28.5,26.2,26.1$, 24.0, 19.8, 16.0; HRMS calcd for $453.2253(\mathrm{M}+\mathrm{Na})$. Found 453.2291. 26: $\mathrm{mp} 146.5-147.5^{\circ} \mathrm{C} ;[\alpha]_{\mathrm{D}}=-4.1(c$ 1.2, $\left.\mathrm{CHCl}_{3}\right)$; IR $\left(\mathrm{cm}^{-1}\right) 3351,1715,1273 ;{ }^{1} \mathrm{H}$ NMR $(300$ $\left.\mathrm{MHz}, \mathrm{CDCl}_{3}\right) \delta 8.06(\mathrm{~d}, J=8.1 \mathrm{~Hz}, 2 \mathrm{H}), 7.58(\mathrm{t}, J=7.5$ $\mathrm{Hz}, 1 \mathrm{H}), 7.46(\mathrm{t}, J=7.5 \mathrm{~Hz}, 2 \mathrm{H}), 5.66(\mathrm{~s}, 2 \mathrm{H}), 5.64$ (s, $1 \mathrm{H}), 5.22(\mathrm{dd}, J=12.3,3.6 \mathrm{~Hz}, 1 \mathrm{H}), 2.97(\mathrm{~d}, J=6.9 \mathrm{~Hz}$, $1 \mathrm{H}), 2.43-2.38(\mathrm{~m}, 2 \mathrm{H}), 2.18(\mathrm{dd}, J=4.5,3.6 \mathrm{~Hz}, 1 \mathrm{H})$, $2.02-1.98(\mathrm{~m}, 1 \mathrm{H}), 1.51(\mathrm{q}, J=12 \mathrm{~Hz}, 1 \mathrm{H}), 1.25(\mathrm{~d}, J=7.5$ $\mathrm{Hz}, 3 \mathrm{H}), 1.24(\mathrm{~s}, 3 \mathrm{H}), 1.23(\mathrm{~s}, 3 \mathrm{H}), 1.17(\mathrm{~s}, 3 \mathrm{H}) ;{ }^{13} \mathrm{C}$ NMR $(75 \mathrm{MHz}) \delta$ 166.4, 146.1, 132.9, 132.2, 130.6, $129.5,128.4,123.8,122.2,79.3,72.4,46.0,39.7,38.9$, 34.9, 27.9, 27.1, 25.9, 24.8, 21.4; HRMS calcd for $363.1936(\mathrm{M}+\mathrm{Na})$, Found 363.1970. 21: $\mathrm{mp} 189-191^{\circ} \mathrm{C}$; $[\alpha]_{\mathrm{D}}=-42.8(c 1.05, \mathrm{MeOH}) ; \mathrm{IR}\left(\mathrm{cm}^{-1}\right)$ 3413, 1696, 1025; ${ }^{1} \mathrm{H}$ NMR $\left(300 \mathrm{MHz}, \mathrm{MeOH}-d_{4}\right) \delta 4.06(\mathrm{~s}, 1 \mathrm{H}), 3.87$ (d, $J=2.7 \mathrm{~Hz}, 1 \mathrm{H}), 3.25-3.17(\mathrm{~m}, 2 \mathrm{H}), 2.67(\mathrm{t}, J=13.5 \mathrm{~Hz}$, $1 \mathrm{H}), 2.34-2.19(\mathrm{~m}, 2 \mathrm{H}), 1.87-1.81(\mathrm{~m}, 2 \mathrm{H}), 1.51(\mathrm{dt}$, $J=13.5,2.4 \mathrm{~Hz}, 1 \mathrm{H}), 1.32(\mathrm{~s}, 3 \mathrm{H}), 1.12(\mathrm{~s}, 3 \mathrm{H}), 0.91$ (d, $J=6.6 \mathrm{~Hz}, 3 \mathrm{H}), 0.54(\mathrm{~s}, 3 \mathrm{H}) ;{ }^{13} \mathrm{C} \mathrm{NMR}(75 \mathrm{MHz}) \delta$ 216.7, 74.2, 73.7, 72.0, 69.7, 47.5, 46.6, 44.9, 38.1, 35.7, 28.8, 28.4, 27.6, 14.0, 10.5; HRMS calcd for 309.1678 $(\mathrm{M}+\mathrm{Na})$. Found 309.1692. 17: $[\alpha]_{\mathrm{D}}=-62.5 \quad(c \quad 1.2$, $\left.\mathrm{CHCl}_{3}\right)$; IR $\left(\mathrm{cm}^{-1}\right) 3479,1717,1275 ;{ }^{1} \mathrm{H}$ NMR $(300$ $\left.\mathrm{MHz}, \mathrm{CDCl}_{3}\right) \delta 8.03(\mathrm{~d}, J=6.9 \mathrm{~Hz}, 2 \mathrm{H}), 7.56(\mathrm{t}, J=6.9$ $\mathrm{Hz}, 1 \mathrm{H}), 7.44$ (t, $J=7.2 \mathrm{~Hz}, 2 \mathrm{H}), 5.67-5.62$ (br s, 2H), $5.24(\mathrm{dd}, J=12,4.5 \mathrm{~Hz}, 1 \mathrm{H}), 3.31(\mathrm{~d}, J=3 \mathrm{~Hz}, 1 \mathrm{H})$, 2.29-1.96 (m, 3H), 1.91-1.87 (m, 2H), 1.79-1.67 (m, 1H), 
$1.43(\mathrm{~s}, 3 \mathrm{H}), 1.40(\mathrm{~s}, 3 \mathrm{H}), 1.30$ (s, 3H), $1.24(\mathrm{~d}, J=7.8 \mathrm{~Hz}$, $3 \mathrm{H}) ;{ }^{13} \mathrm{C}$ NMR $(75 \mathrm{MHz}) \delta 165.9,132.9,130.6,129.5$, 128.4, 123.4, 76.6, 74.2, 66.3, 58.9, 41.8, 39.0, 35.9, 34.2, 27.9, 27.7, 23.7, 18.5, 16.5; HRMS calcd for 379.1885 $(\mathrm{M}+\mathrm{Na})$. Found 379.1907. 16: $[\alpha]_{\mathrm{D}}=-33.1 \quad(c=1.0$, $\left.\mathrm{CHCl}_{3}\right)$; IR $\left(\mathrm{cm}^{-1}\right) 3489,1715,1275 ;{ }^{1} \mathrm{H}$ NMR (300 $\left.\mathrm{MHz}, \mathrm{CDCl}_{3}\right) \delta 8.06(\mathrm{~d}, J=7.5 \mathrm{~Hz}, 2 \mathrm{H}), 7.56(\mathrm{~d}, J=6.6$ $\mathrm{Hz}, 1 \mathrm{H}), 7.45(\mathrm{t}, J=6.6 \mathrm{~Hz}, 2 \mathrm{H}), 5.66(\mathrm{~s}, 1 \mathrm{H}), 5.60(\mathrm{~s}$, $2 \mathrm{H}), 5.16(\mathrm{t}, J=6.9 \mathrm{~Hz}, 1 \mathrm{H}), 2.96(\mathrm{~d}, J=6.3 \mathrm{~Hz}, 1 \mathrm{H})$, 2.38 (br s, 1H), 2.17-2.12 (m, 1H), 1.99-1.95 (m, 3H), $1.31(\mathrm{~s}, 6 \mathrm{H}), 1.27(\mathrm{~s}, 3 \mathrm{H}), 1.25(\mathrm{~d}, J=7.5 \mathrm{~Hz}, 3 \mathrm{H}) ;{ }^{13} \mathrm{C}$ NMR $(75 \mathrm{MHz}) \delta 166.3,145.8,132.8,131.5,129.5$, $128.4,122.8,121.7,78.0,73.3,44.8,37.8,37.6,37.0,27.9$, 27.6, 25.6, 24.2, 21.0; HRMS calcd for $363.1936(\mathrm{M}+\mathrm{Na})$. Found 363.1956. 13: $[\alpha]_{\mathrm{D}}=-66.2\left(\right.$ c $\left.0.8, \mathrm{CHCl}_{3}\right)$; IR $\left(\mathrm{cm}^{-1}\right) 3321,1455 ;{ }^{1} \mathrm{H}$ NMR $\left(300 \mathrm{MHz}, \mathrm{CDCl}_{3}\right) \delta 5.63-$ $5.60(\mathrm{~m}, 1 \mathrm{H}), 5.48-5.46(\mathrm{~m}, 1 \mathrm{H}), 4.73(\mathrm{~s}, 2 \mathrm{H}), 3.59-3.55$ (m, 1H), 3.14-3.07 (br s, -OH), 2.75-2.72 (m, 1H), 2.18$2.12(\mathrm{~m}, 2 \mathrm{H}), 1.81-1.63(\mathrm{~m}, 4 \mathrm{H}), 1.74(\mathrm{~s}, 3 \mathrm{H}), 1.46(\mathrm{dd}$, $J=8.7,3.3 \mathrm{~Hz}, 1 \mathrm{H}), 1.21(\mathrm{~s}, 3 \mathrm{H}), 1.15(\mathrm{~d}, J=7.5 \mathrm{~Hz}, 3 \mathrm{H})$; ${ }^{13} \mathrm{C}$ NMR $(75 \mathrm{MHz}) \delta 149.2,131.4,121.0,109.0,78.5$, 75.7, 43.5, 42.2, 39.3, 35.5, 34.0, 33.9, 21.1, 21.0, 16.0; HRMS calcd for $259.1674(\mathrm{M}+\mathrm{Na})$. Found 259.1695. 12: $[\alpha]_{\mathrm{D}}=+75.9\left(c=0.83, \mathrm{CHCl}_{3}\right) ; \mathrm{IR}\left(\mathrm{cm}^{-1}\right) 3343,1455 ;{ }^{1} \mathrm{H}$ NMR $\left(300 \mathrm{MHz}, \mathrm{CDCl}_{3}\right) \delta 5.47(\mathrm{br} \mathrm{s}, 1 \mathrm{H}), 5.36(\mathrm{~d}$, $J=9.9 \mathrm{~Hz}, 1 \mathrm{H}), 4.74(\mathrm{~s}, 2 \mathrm{H}), 3.63($ br s, $1 \mathrm{H}), 3.09$ (d, $J=6.6 \mathrm{~Hz}, 1 \mathrm{H}), 2.97(\mathrm{~s}, 1 \mathrm{H}), 2.68-2.65(\mathrm{~m}, 1 \mathrm{H}), 2.45(\mathrm{~m}$, $1 \mathrm{H}), 2.18(\mathrm{~d}, J=17.7 \mathrm{~Hz}, 1 \mathrm{H}), 1.84-1.81(\mathrm{~m}, 2 \mathrm{H}), 1.75(\mathrm{~s}$, $3 \mathrm{H}), 1.31-1.23(\mathrm{~m}, 1 \mathrm{H}), 1.16(\mathrm{~s}, 3 \mathrm{H}), 0.99(\mathrm{~d}, J=7.5 \mathrm{~Hz}$, $3 \mathrm{H}) ;{ }^{13} \mathrm{C}$ NMR $(75 \mathrm{MHz}) \delta 149.5,130.6,122.8,109.1$, 77.4, 76.1, 39.2, 38.5, 36.1, 33.9, 33.5, 31.9, 20.9, 18.8, 13.2; HRMS calcd for $259.1674(\mathrm{M}+\mathrm{Na})$. Found 259.1683

10. X-Ray crystal structure determination: X-ray data were collected at $293 \mathrm{~K}$ on a SMART CCD-BRUKER diffractometer with graphite monochromated $\mathrm{MoK} \alpha$ radiation $(\lambda=0.7107 \AA)$. Structure was solved by direct methods (SIR92). Refinement was by full-matrix leastsquares procedures on $F^{2}$ using SHELXL-97. The nonhydrogen atoms were refined anisotropically whereas hydrogen atoms were refined isotropically. Compound PNB derivative of 12: $\mathrm{C}_{22} \mathrm{H}_{27} \mathrm{O}_{5} \mathrm{~N}, \mathrm{MW}=385.45$, crystal system: monoclinic, space group: $P 2(1)$, cell parameters: $a=8.265 \quad$ (2), $\quad b=13.123 \quad$ (3), $\quad c=18.829(5) \quad \AA, \quad \beta=$ 94.113(5), $V=2037.09(6) \AA^{3}, Z=4, D_{\text {calcd }}=1.257 \mathrm{~g} \mathrm{~cm}^{-3}$, $F(000)=824.0, \quad \mu=0.09 \mathrm{~mm}^{-1}$. Total number of 1.s. parameters $=721, R_{1}=0.0576$ for $6673, F_{\mathrm{o}}>4 \sigma\left(F_{\mathrm{o}}\right)$ and 0.0797 for all 8820 data. $W R_{2}=0.1213, \mathrm{GOF}=1.123$. Restrained $\mathrm{GOF}=1.123$ for all data. There are two molecules in an asymmetric unit. An ORTEP diagram with only one molecule is shown in Scheme 2. (CCDC 214302). Compound 21: $\mathrm{C}_{15} \mathrm{H}_{26} \mathrm{O}_{5}, \mathrm{MW}=286.37$, colorless crystal, Crystal system: monoclinic, space group: $P 2$ (1), cell parameters: $a=7.6151(6), b=12.361(1), c=$ $16.356(1) \AA, V=1539.76 \AA^{3}, Z=4, D_{\text {calcd }}=1.235 \mathrm{~g} \mathrm{~cm}^{-3}$, $F(000)=624.0, \quad \mu=0.09 \mathrm{~mm}^{-1}$. Total number of 1.s. parameters $=285, R_{1}=0.0385$ for $2927, F_{\mathrm{o}}>4 \sigma\left(F_{\mathrm{o}}\right)$ and 0.0417 for all 53125 data. $W R_{2}=0.0925, \mathrm{GOF}=1.086$. Restrained $\mathrm{GOF}=1.086$ for all data (CCDC 214303).

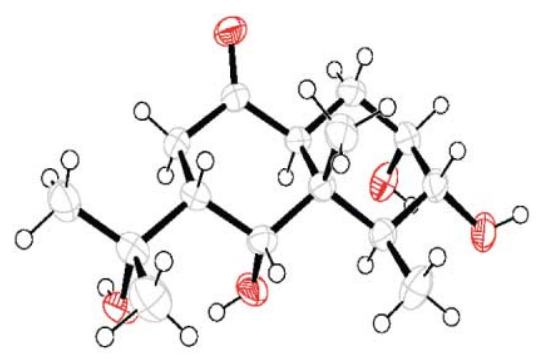

ORTEP diagram of $\mathbf{2 1}$

Compound 22: $\mathrm{C}_{15} \mathrm{H}_{22} \mathrm{O}_{2}, \mathrm{MW}=234.34$, colorless crystal, crystal system: monoclinic, space group: $P 2(1)$, cell parameters: $a=10.305(2), b=10.916(2), c=12.805(3) \AA$, $\beta=108.436(4), V=1366.75(6) \AA^{3}, Z=4, D_{\text {calcd }}=1.139 \mathrm{~g}$ $\mathrm{cm}^{-3}, F(000)=512.0, \mu=0.07 \mathrm{~mm}^{-1}$. Total number of 1.s. parameters $=315, R_{1}=0.0565$ for $4301, F_{\mathrm{o}}>4 \sigma\left(F_{\mathrm{o}}\right)$ and 0.0739 for all 5368 data. $\mathrm{WR} 2=0.1254, \mathrm{GOF}=1.106$, restrained $\mathrm{GOF}=1.106$ for all data. There are two molecules in an asymmetric unit. (CCDC 214304).

Compound 29: $\mathrm{C}_{22} \mathrm{H}_{30} \mathrm{O}_{6}, \mathrm{MW}=390.48$, crystal system: monoclinic, space group: $P 2(1)$, cell parameters: $a=$ 8.198(2), $b=15.354(4), c=16.138$ (4) $\AA, \beta=94.364$ (5), $V=2025.55 \AA^{3}, Z=4, D_{\text {calcd }}=1.28 \mathrm{~g} \mathrm{~cm}^{-3}, \quad F(000)=$ $840.0, \mu=0.09 \mathrm{~mm}^{-1}$. Total number of 1.s. parameters $=$ $239, R_{1}=0.0897$ for $5614, F_{\mathrm{o}}>4 \sigma\left(F_{\mathrm{o}}\right)$ and 0.1226 for all 7976 data. $w R_{2}=0.2182, \quad \mathrm{GOF}=1.025$, Restrained $\mathrm{GOF}=1.025$ for all data. There are two molecules in an asymmetric unit. (CCDC 214305).

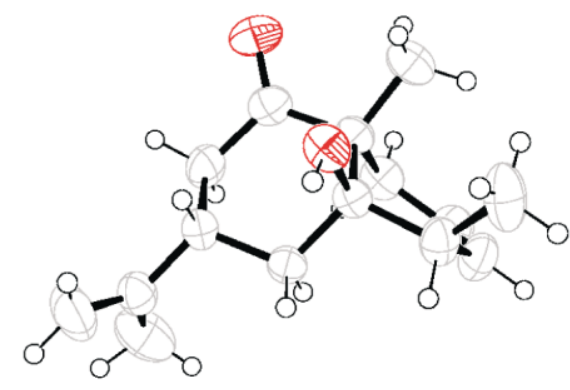

ORTEP diagram of $\mathbf{2 2}$

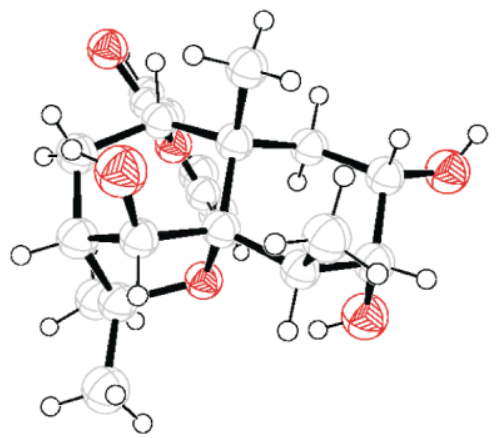

ORTEP diagram of $\mathbf{2 9}$ 